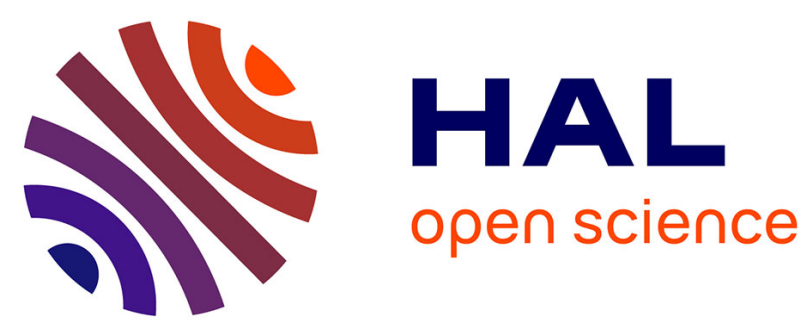

\title{
Local and Full-cycle Mobility Analysis of a 3-RPS-3-SPR Series-Parallel Manipulator
}

\author{
Abhilash Nayak, Stéphane Caro, Philippe Wenger
}

\section{To cite this version:}

Abhilash Nayak, Stéphane Caro, Philippe Wenger. Local and Full-cycle Mobility Analysis of a 3RPS-3-SPR Series-Parallel Manipulator. 7th IFToMM International Workshop on Computational Kinematics (CK2017), May 2017, Poitiers, France. hal-01757936

\section{HAL Id: hal-01757936 https://hal.science/hal-01757936}

Submitted on 4 Apr 2018

HAL is a multi-disciplinary open access archive for the deposit and dissemination of scientific research documents, whether they are published or not. The documents may come from teaching and research institutions in France or abroad, or from public or private research centers.
L'archive ouverte pluridisciplinaire HAL, est destinée au dépôt et à la diffusion de documents scientifiques de niveau recherche, publiés ou non, émanant des établissements d'enseignement et de recherche français ou étrangers, des laboratoires publics ou privés. 


\title{
Local and Full-cycle Mobility Analysis of a 3-RPS-3-SPR Series-Parallel Manipulator
}

\author{
Abhilash Nayak $^{1}$, Stéphane Caro ${ }^{2}$, Philippe Wenger ${ }^{2}$ \\ ${ }^{1}$ École Centrale de Nantes, Laboratoire des Sciences du Numérique de \\ Nantes (LS2N), 1 rue de la Noë, 44321 Nantes, France. e-mail: \\ Abhilash.Nayak@irccyn.ec-nantes.fr \\ ${ }^{2}$ CNRS, Laboratoire des Sciences du Numrique de Nantes (LS2N), École Centrale \\ de Nantes, 1 rue de la Nö̈, UMR CNRS 6004, 44321 Nantes, France e-mail: \\ \{Stephane.Caro, Philippe.Wenger\}@ls2n.fr
}

\begin{abstract}
This paper aims to shed light on the determination of the true mobility for a 3-RPS-3SPR series-parallel manipulator, which was claimed to be six in [6] without any proof, and shown to be five in [4] and [3] with an erroneous proof. Screw theory is used to derive the kinematic Jacobian matrix and the twist system of the mechanism, leading to the determination of its local mobility. I turns out that this local mobility is found to be six in several arbitrary configurations, which indicates a full-cycle mobility equal to six. This full-cycle mobility is confirmed by calculating the Hilbert dimension of the ideal made up of the set of constraint equations. It is also shown that the mobility drops to five in some particular configurations, referred to as impossible output singularities.
\end{abstract}

Key words: series-parallel manipulator, mobility analysis, Jacobian matrix, screw theory, Hilbert dimension

\section{Introduction}

A series-parallel manipulator (S-PM) is composed of parallel manipulators mounted in series and has merits of both serial and parallel manipulators. The 3-R $\underline{P S}-3-\mathrm{S} \underline{\mathrm{PR}}$ $\mathrm{S}-\mathrm{PM}$ is such a mechanism with the proximal module being composed of the 3-RPS parallel mechanism and the distal module being composed of the 3-SPR PM. Hu et al. [6] analyzed the workspace of this manipulator. Hu formulated the Jacobian matrix for S-PMs as a function of Jacobians of the individual parallel modules [5]. In the former paper, it was assumed that the number of local dof of the 3-RPS-3-SPR mechanism is equal to six, whereas Gallardo et al. found out that it is equal to five [4, 3]. As a matter of fact, it is not straightforward to find the local mobility of this S-PM due to the third-order twist systems of each individual module. It is established that the 3-RPS PM performs a translation and two non pure rotations about non fixed axes, which induce two translational parasitic motions [7]. The 3-SPR PM also has the same type of dof [12]. In addition, these mechanisms are known as zero-torsion mechanisms. When they are mounted in series, the axis about which the torsional motion is constrained, is different for a general configuration of the S-PM. Gallardo et al. failed to consider this fact but only those special configurations in which the 
axes coincide resulting in a mobility equal to five. This paper aims at clarifying that the full-cycle mobility of the 3-RPS-3-SPR S-PM is equal to six with the help of screw theory and some algebraic geometry concepts. Although the considered SPM has double spherical joints and two sets of three coplanar revolute joint axes, the proposed methodology to calculate the mobility of the manipulator at hand is general and can be applied to any series-parallel manipulator.

The paper is organized as follows : The manipulator under study is described in Section 2. The kinematic Jacobian matrix of a general S-PM with multiple modules is expressed in vector form in Section 3. Section 4 presents some configurations of the 3-RPS-3-SPR S-PM with the corresponding local mobility. Section 5 deals with the full-cycle mobility of the 3-RPS-3-SPR S-PM.

\section{Manipulator under study}

The architecture of the 3-RPS-3-SPR S-PM under study is shown in Fig. 1. It consists of a proximal 3-RPS PM module and a distal 3-SPR PM module. The 3-RPS $\mathrm{PM}$ is composed of three legs each containing a revolute, a prismatic and a spherical joint mounted in series, while the legs of the 3-SPR PM have these lower pairs in reverse order. Thus, the three equilateral triangular shaped platforms are the fixed base, the coupler and the end effector, coloured brown, green and blue, respectively. The vertices of these platforms are named $A_{i}, B_{i}$ and $C_{i}, i=0,1,2$. Here after, the subscript 0 corresponds to the fixed base, 1 to the coupler platform and 2 to the end-effector. A coordinate frame $\mathscr{F}_{i}$ is attached to each platform such that its origin $O_{i}$ lies at its circumcenter. The coordinate axes, $x_{i}$ points towards the vertex $A_{i}, y_{i}$ is parallel to the opposite side $B_{i} C_{i}$ and by the right hand rule, $z_{i}$ is normal to platform plane. Besides, the circum-radius of the $i$-th platform is denoted as $h_{i}$. $\mathbf{p}_{i}$ and $\mathbf{q}_{i}$, $i=1, \ldots, 6$ are unit vectors along the prismatic joints while $\mathbf{u}_{i}$ and $\mathbf{v}_{i}, i=1, \ldots, 6$ are unit vectors along the revolute joint axes.

\section{Kinematic modeling of series-parallel manipulators}

Keeping in mind that the two parallel mechanisms are mounted in series, the end effector twist (angular velocity vector of a body and linear velocity vector of a point

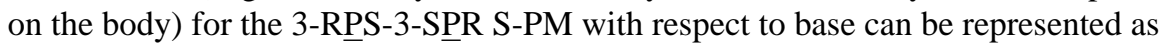
follows:

$$
{ }^{0} \mathbf{t}_{2 / 0}={ }^{0} \mathbf{t}_{2 / 0}^{\text {PROX }}+{ }^{0} \mathbf{t}_{2 / 1}^{\text {DIST }} \Longrightarrow\left[\begin{array}{c}
{ }^{0} \omega_{2 / 0} \\
{ }^{0} \mathbf{v}_{O_{2} / 0}
\end{array}\right]=\left[\begin{array}{c}
{ }^{0} \omega_{2 / 0}^{\text {PROX }} \\
{ }^{0} \mathbf{v}_{O_{2} / 0}^{R O X}
\end{array}\right]+\left[\begin{array}{c}
{ }^{0} \omega_{2 / 1}^{D I S T} \\
{ }^{0} \mathbf{v}_{O_{2} / 1}^{D I T}
\end{array}\right]
$$

where ${ }^{0} \mathbf{t}_{2 / 0}^{P R O X}$ is the end effector twist with respect to the base (2/0) due to the proximal module motion and ${ }^{0} \mathbf{t}_{2 / 1}^{D I S T}$ is the end effector twist with respect to the coupler 


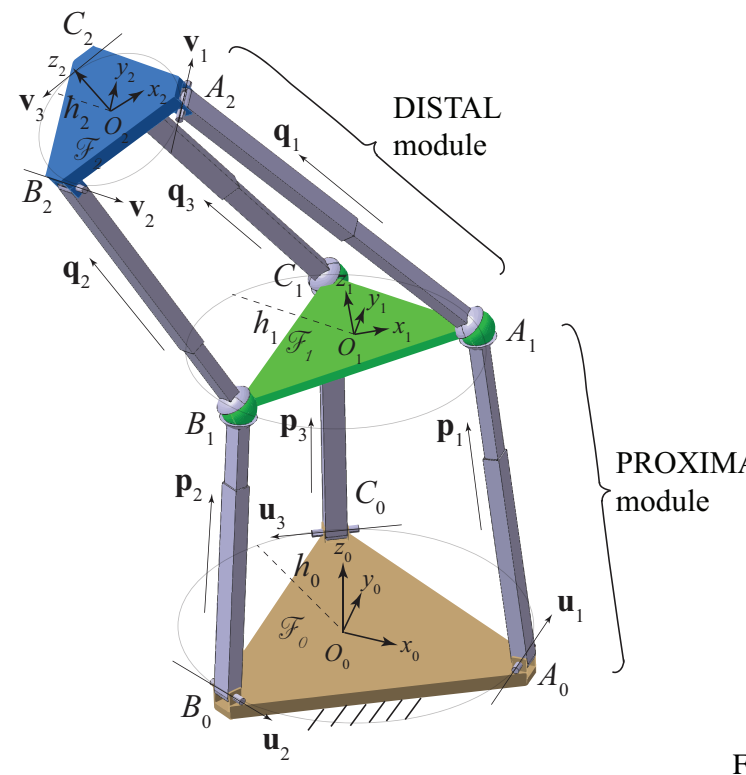

Fig. 1: A 3-R $\underline{P S}-3-S \underline{P R}$ series-parallel manipulator

Fig. 2: $n$ parallel mechanisms (named modules) arranged in series

$(2 / 1)$ due to the distal module motion. These twists are expressed in the base frame $\mathscr{F} 0$, hence the left superscript. The terms on right hand side of Eq. (1) are not known, but can be expressed in terms of the known twists using screw transformations. To do so, the known twists are first noted down. If the proximal and distal modules are considered individually, the twist of their respective moving platforms with respect to their fixed base will be expressed as a function of the actuated joint velocities :

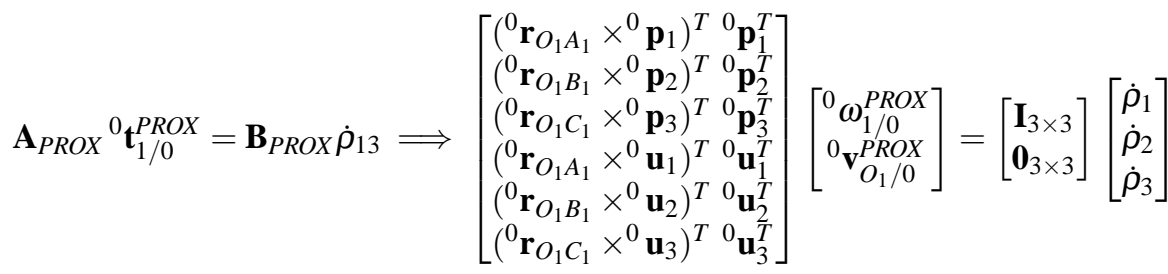

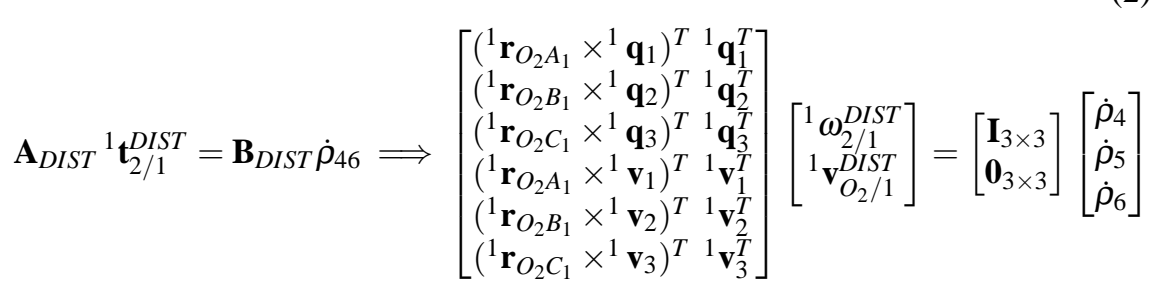


where, ${ }^{0} \mathbf{t}_{1 / 0}^{P R O X}$ is the twist of the coupler with respect to the base expressed in $\mathscr{F}_{0}$ and ${ }^{1} \mathbf{t}_{2 / 1}^{D I S T}$ is the twist of the end effector with respect to the coupler expressed in $\mathscr{F}_{1}$. $\mathbf{A}_{P R O X}$ and $\mathbf{A}_{D I S T}$ are called forward Jacobian matrices and they incorporate the actuation and constraint wrenches of the 3-RPS and 3-SPR PMs, respectively [9]. $\mathbf{B}_{P R O X}$ and $\mathbf{B}_{\text {DIST }}$ are called inverse Jacobian matrices and they are the result of the reciprocal product between wrenches of the mechanism and twists of the joints for the 3-RPS and 3-SPR PMs, respectively. $\dot{\rho}_{13}=\left[\dot{\rho}_{1}, \dot{\rho}_{2}, \dot{\rho}_{3}\right]^{T}$ and $\dot{\rho}_{46}=\left[\dot{\rho}_{4}, \dot{\rho}_{5}, \dot{\rho}_{6}\right]^{T}$ are the prismatic joint velocities for the proximal and distal modules, respectively. ${ }^{k} \mathbf{r}_{P Q}$ denotes the vector pointing from a point $P$ to point $Q$ expressed in frame $\mathscr{F}_{k}$. Considering Eq. (1), the unknown twists ${ }^{0} \mathbf{t}_{2 / 0}^{P R O X}$ and ${ }^{0} \mathbf{t}_{2 / 1}^{D I S T}$ can be expressed in terms of the known twists ${ }^{0} \mathbf{t}_{1 / 0}^{P R O X}$ and ${ }^{1} \mathbf{t}_{2 / 1}^{P R O X}$ using the following screw transformation matrices $[11,2]$.

$$
\begin{aligned}
& {\left[\begin{array}{c}
{ }^{0} \omega_{2 / 0}^{P R O X} \\
{ }^{0} \mathbf{v}_{O_{2} / 0}^{P R O X}
\end{array}\right]={ }^{2} \mathbf{A d}_{1}\left[\begin{array}{c}
{ }^{0} \omega_{1 / 0}^{P R O X} \\
{ }^{0} \mathbf{v}_{O_{1} / 0}^{P R O X}
\end{array}\right]} \\
& \text { with }{ }^{2} \mathbf{A d} \mathbf{d}_{1}=\left[\begin{array}{cc}
\mathbf{I}_{3 \times 3} & \mathbf{0}_{3 \times 3} \\
-{ }^{0} \hat{\mathbf{r}}_{O_{1} O_{2}} & \mathbf{I}_{3 \times 3}
\end{array}\right], \quad{ }^{0} \hat{\mathbf{r}}_{O_{1} O_{2}}=\left[\begin{array}{ccc}
0 & -{ }^{0} z_{O_{1} O_{2}} & { }^{0} y_{O_{1} O_{2}} \\
{ }^{0} z_{O_{1} O_{2}} & 0 & -{ }^{0} x_{O_{1} O_{2}} \\
-{ }^{0} y_{O_{1} O_{2}} & { }^{0} x_{O_{1} O_{2}} & 0
\end{array}\right]
\end{aligned}
$$

${ }^{2} \mathbf{A d}_{1}$ is called the adjoint matrix. ${ }^{0} \hat{\mathbf{r}}_{O_{1} O_{2}}$ is the cross product matrix of vector ${ }^{0} \mathbf{r}_{O_{1} O_{2}}=\left[{ }^{0} x_{O_{1} O_{2}},{ }^{0} y_{O_{1} O_{2}},{ }^{0} z_{O_{1} O_{2}}\right]$, pointing from point $O_{1}$ to point $O_{2}$ expressed in frame $\mathscr{F}_{0}$.

Similarly, for the distal module, the velocities ${ }^{1} \omega_{2 / 1}^{D I S T}$ and ${ }^{1} \mathbf{v}_{O_{2} / 1}^{D I S T}$ can be transformed from frame $\mathscr{F}_{1}$ to $\mathscr{F}_{0}$ just by multiplying each of them by the rotation matrix ${ }^{0} \mathbf{R}_{1}$ from frame $\mathscr{F}_{0}$ to frame $\mathscr{F}_{1}$ :

$$
\left[\begin{array}{c}
{ }^{0} \omega_{2 / 1}^{D I S T} \\
{ }^{0} \mathbf{v}_{O_{2} / 1}^{D I S T}
\end{array}\right]={ }^{0} \overline{\mathbf{R}}_{1}\left[\begin{array}{c}
{ }^{1} \omega_{2 / 1}^{D I S T} \\
{ }^{1} \mathbf{v}_{O_{2} / 1}^{D I S T}
\end{array}\right] \text { with }{ }^{0} \overline{\mathbf{R}}_{1}=\left[\begin{array}{cc}
{ }^{0} \mathbf{R}_{1} & \mathbf{I}_{3 \times 3} \\
\mathbf{I}_{3 \times 3} & { }^{0} \mathbf{R}_{1}
\end{array}\right]
$$

${ }^{0} \overline{\mathbf{R}}_{1}$ is called the augmented rotation matrix between frames $\mathscr{F}_{0}$ and $\mathscr{F}_{1}$. Consequently from Eqs. (4) and (5),

$$
{ }^{0} \mathbf{t}_{2 / 0}={ }^{2} \mathbf{A d}_{1}{ }^{0} \mathbf{t}_{1 / 0}^{P R O X}+{ }^{0} \overline{\mathbf{R}}_{1}{ }^{1} \mathbf{t}_{2 / 1}^{D I S T}
$$

Note that Eq. (6) amounts to the twist equation derived in [5] whereas Gallardo et al. add the twists of individual modules directly without considering the screw transformations. It is noteworthy that Equation (11) in [4] is incorrect, so are any further conclusions based on this equation. Following Eqs. (2) and (3), with the assumption that the proximal and distal modules are not in a parallel singularity ${ }^{1}$ or in other words, matrices $\mathbf{A}_{P R O X}$ and $\mathbf{A}_{D I S T}$ are invertible,

\footnotetext{
${ }^{1}$ Parallel singularity can be an actuation singularity, constraint singularity or a compound singularity $[13,10,1]$
} 


$$
\begin{aligned}
& { }^{0} \mathbf{t}_{2 / 0}={ }^{2} \mathbf{A d}_{1} \mathbf{A}_{\text {PROX }}^{-1} \mathbf{B}_{\text {PROX }} \dot{\rho}_{13}+{ }^{0} \overline{\mathbf{R}}_{1} \mathbf{A}_{\text {DIST }}^{-1} \mathbf{B}_{\text {DIST }} \dot{\rho}_{46} \\
& =\left[\begin{array}{ll}
{ }^{2} \mathbf{A d}_{1} \mathbf{A}_{P R O X}^{-1} \mathbf{B}_{P R O X} & { }^{0} \overline{\mathbf{R}}_{1} \mathbf{A}_{D I S T}^{-1} \mathbf{B}_{D I S T}
\end{array}\right]\left[\begin{array}{l}
\dot{\rho}_{13} \\
\dot{\rho}_{46}
\end{array}\right] \\
& =\mathbf{J}_{\mathrm{S}-\mathrm{PM}}\left[\begin{array}{c}
\dot{\rho}_{13} \\
\dot{\rho}_{46}
\end{array}\right]
\end{aligned}
$$

$\mathbf{J}_{\mathrm{S}-\mathrm{PM}}$ is the kinematic Jacobian matrix of the 3-RPS-3-SPR S-PM under study. The rank of this matrix provides the local mobility of the S-PM.

Equations (6), (7) and (8) can be extended to a series-parallel manipulator with $n$ number of parallel mechanisms, named modules in this paper, in series as shown in Fig. 2. Thus, the twist of the end effector with respect to the fixed base expressed in frame $\mathscr{F}_{0}$ can be expressed as follows :

$$
\begin{aligned}
& { }^{0} \mathbf{t}_{n / 0}=\sum_{i=1}^{n}{ }^{0} \overline{\mathbf{R}}_{(i-1)}{ }^{n} A d_{i}{ }^{(i-1)} \mathbf{t}^{M_{i}}{ }^{M_{(i-1)}}=\mathbf{J}_{6 \times 3 n}\left[\begin{array}{c}
\dot{\rho}_{M_{1}} \\
\dot{\rho}_{M_{2}} \\
\vdots \\
\dot{\rho}_{M_{n}}
\end{array}\right] \\
& \text { with } \quad{ }^{0} \overline{\mathbf{R}}_{i}=\left[\begin{array}{cc}
{ }^{0} \mathbf{R}_{i} & \mathbf{I}_{3 \times 3} \\
\mathbf{I}_{3 \times 3} & { }^{0} \mathbf{R}_{i}
\end{array}\right], \quad{ }^{n} \mathbf{A d}_{i}=\left[\begin{array}{cc}
\mathbf{I}_{3 \times 3} & \mathbf{0}_{3 \times 3} \\
-{ }^{(i-1)} \hat{\mathbf{r}}_{O_{i} O_{n}} & \mathbf{I}_{3 \times 3}
\end{array}\right] \text { and } \\
& \mathbf{J}_{6 \times 3 n}=\left[\begin{array}{lllll}
{ }^{n} \mathbf{A d}_{1} \mathbf{A}_{M_{0}}^{-1} \mathbf{B}_{M_{0}} & { }^{0} \overline{\mathbf{R}}_{1}{ }^{n} \mathbf{A d}_{2} \mathbf{A}_{M_{1}}^{-1} \mathbf{B}_{M_{1}} & \ldots & { }^{0} \overline{\mathbf{R}}_{n} \mathbf{A}_{M_{n}}^{-1} \mathbf{B}_{M_{n}}
\end{array}\right]
\end{aligned}
$$

where, $\mathbf{J}_{6 \times 3 n}$ is the $6 \times 3 n$ kinematic Jacobian matrix of the $n$-module hybrid manipulator. $M_{i}$ stands for the $i$-th module, $\mathbf{A}_{M_{i}}$ and $\mathbf{B}_{M_{i}}$ are the forward and inverse Jacobian matrices of $M_{i}$ of the series-parallel manipulator, respectively. $\dot{\rho}_{M_{i}}$ is the vector of the actuated prismatic joint rates for the $i$-th module.

\section{Twist system of the 3-RPS-3-SPR S-PM}

Each leg of the 3-RPS and 3-SPR parallel manipulators are composed of three joints, but the order of the limb twist system is equal to five and hence there exist five twists associated to each leg. Thus, the constraint wrench system of the $i$-th leg reciprocal to the foregoing twists is spanned by a pure force $\mathscr{W}^{i}$ passing through the spherical joint center and parallel to the revolute joint axis. Therefore, the constraint wrench systems of the proximal and distal modules are spanned by three zero-pitch wrenches, namely,

$$
\begin{aligned}
& { }^{0} \mathscr{W}_{\text {PROX }}=\bigoplus_{i=1}^{3}{ }^{0} \mathscr{W}_{\text {PROX }}^{i}=\operatorname{span}\left\{\left[\begin{array}{c}
{ }^{0} \mathbf{u}_{1} \\
{ }^{0} \mathbf{r}_{O_{2} A_{1}} \times{ }^{0} \mathbf{u}_{1}
\end{array}\right],\left[\begin{array}{c}
{ }^{0} \mathbf{u}_{2} \\
{ }^{0} \mathbf{r}_{O_{2} B_{1}} \times{ }^{0} \mathbf{u}_{2}
\end{array}\right],\left[\begin{array}{c}
{ }^{0} \mathbf{u}_{3} \\
{ }^{0} \mathbf{r}_{O_{2} C_{1}} \times{ }^{0} \mathbf{u}_{3}
\end{array}\right]\right\} \\
& { }^{1} \mathscr{W}_{\text {DIST }}=\bigoplus_{i=1}^{3}{ }^{1} \mathscr{W}_{\text {DIST }}^{i}=\operatorname{span}\left\{\left[\begin{array}{c}
{ }^{1} \mathbf{v}_{1} \\
{ }^{1} \mathbf{r}_{O_{2} A_{1}} \times{ }^{1} \mathbf{v}_{1}
\end{array}\right],\left[\begin{array}{c}
{ }^{1} \mathbf{v}_{2} \\
{ }^{1} \mathbf{r}_{O_{2} B_{1}} \times{ }^{1} \mathbf{v}_{2}
\end{array}\right],\left[\begin{array}{c}
{ }^{1} \mathbf{v}_{3} \\
{ }^{1} \mathbf{r}_{O_{2} C_{1}} \times{ }^{1} \mathbf{v}_{3}
\end{array}\right]\right\}
\end{aligned}
$$


Due to the serial arrangement of the parallel mechanisms, the constraint wrench system of the S-PM is the intersection of the constraint wrench systems of each module. Alternatively, the twist system of the S-PM is the direct sum (disjoint union) of the twist systems of each module. Therefore, the nullspace of the $3 \times 6$ matrix containing the basis screws of ${ }^{0} \mathscr{W}_{P R O X}$ and ${ }^{1} \mathscr{W}_{\text {DIST }}$ leads to the screws that form the basis of the twist system of each module, ${ }^{0} \mathscr{T}_{P R O X}=\operatorname{span}\left\{{ }^{0} \xi_{1},{ }^{0} \xi_{2},{ }^{0} \xi_{3}\right\}$ and ${ }^{1} \mathscr{T}_{\text {DIST }}=\operatorname{span}\left\{{ }^{1} \xi_{4},{ }^{1} \xi_{5},{ }^{1} \xi_{6}\right\}$, respectively. The augmented rotation matrix derived in Eq. (5) is exploited to ensure that all the screws are expressed in one frame $\left(\mathscr{F}_{0}\right.$ in this case). Therefore, the total twist system of the S-PM can be obtained as follows :

$$
\begin{aligned}
{ }^{0} \mathscr{T}_{\mathrm{S}-\mathrm{PM}} & ={ }^{0} \mathscr{T}_{\text {PROX }} \bigoplus^{0} \mathscr{T}_{\text {DIST }} \\
& =\operatorname{span}\left\{{ }^{0} \xi_{1},{ }^{0} \xi_{2},{ }^{0} \xi_{3},{ }^{0} \overline{\mathbf{R}}_{1}{ }^{1} \xi_{4},{ }^{0} \overline{\mathbf{R}}_{1}{ }^{1} \xi_{5},{ }^{0} \overline{\mathbf{R}}_{1}{ }^{1} \xi_{6}\right\}
\end{aligned}
$$

The order of the twist system ${ }^{0} \mathscr{T}_{\mathrm{S} \text {-PM }}$ yields the local mobility of the whole manipulator.

Some general and singular configurations of the 3-RPS-3-SPR S-PM with $h_{0}=2$, $h_{1}=1$ and $h_{2}=2$ are considered and its mobility is listed based on the rank of the Jacobian and the order of the twist system in Table 1. For general configurations like 2 and 3, the mobility is found to be six. The mobility reduces only when some singularities are encountered. For a special configuration when the three platform planes are parallel to each other as shown in the first row of this table, the rotations of the coupler generate translational motions of the end effector. Yet, the torsional axes of both mechanisms coincide and hence, the mechanism cannot perform any rotation about an axis of vertical direction leading to a mobility equal to five. Moreover, a configuration in which any revolute joint axis in the end effector is parallel to its corresponding axis in the fixed base results in a mobility lower than six for the S-PM. For instance, for the 4th configuration in the table, there exists a constraint force $f$, parallel to the two parallel revolute joint axes resulting in a five dof manipulator locally. Configurations 1 and 4 are the impossible output singularities as identified by Zlatanov et al. [15]. It should be noted that if one of the modules is in a parallel singularity, the motion of the moving-platform of the manipulator becomes uncontrollable. A detailed singularity analysis of series-parallel manipulators will be performed in a future work for a better understanding of their behaviour in singular configurations.

\section{Full-cycle mobility of the 3-RPS-3-SPR S-PM}

The full cycle mobility can be obtained by calculating the Hilbert dimension of the set of constraint equations of the mechanism [8]. Two Study transformation matrices are considered : ${ }^{0} \mathbf{X}_{1}$ from $\mathscr{F}_{0}$ to $\mathscr{F}_{1}$ and ${ }^{1} \mathbf{Y}_{2}$ from $\mathscr{F}_{1}$ to $\mathscr{F}_{2}$ composed of Study parameters $x_{i}$ and $y_{i}, i=0,1, \ldots, 7$, respectively. Thus, the coordinates of points $A_{j}, B_{j}$ and $C_{j}, j=0,1,2$ and vectors $\mathbf{u}_{k}$ and $\mathbf{v}_{k}, k=1,2,3$ can be represented in $\mathscr{F}_{0}$ to yield sixteen constraint equations (six for the 3-RPS PM, six for the 3-SPR 


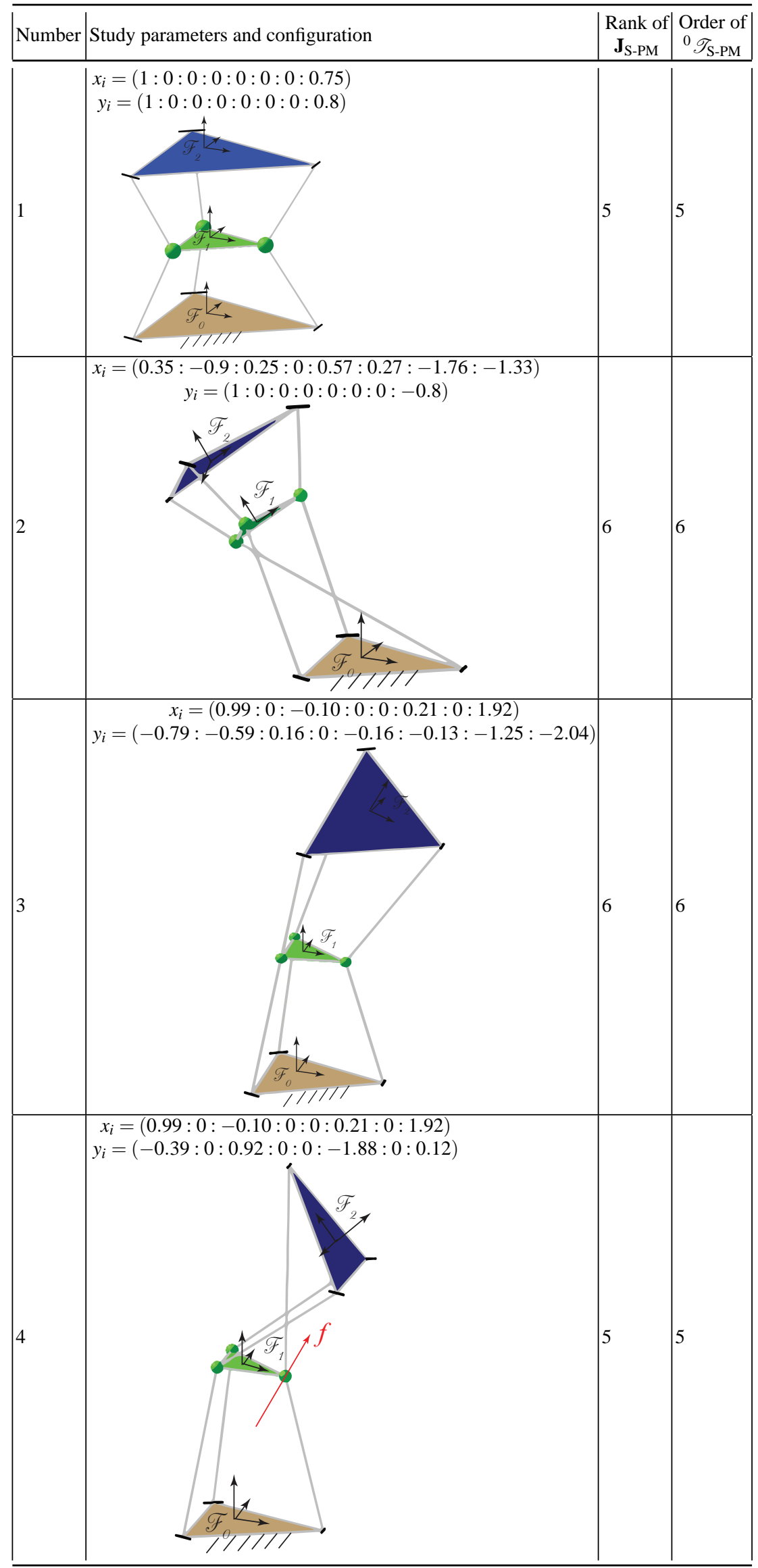

Table 1: Mobility of the 3-RPS-3-SPR S-PM in different configurations 
PM, Study quadric and normalization equations for each transformations). It was established that the 3-RPS and the 3-SPR parallel mechanisms have two operation modes each, characterized by $x_{0}=0, x_{3}=0$ and $y_{0}=0, y_{3}=0$, respectively $[14,12]$. For the S-PM, four ideals of the constraint equations are considered : $\mathscr{K}_{1}$, when $x_{0}=y_{0}=0, \mathscr{K}_{2}$, when $x_{3}=y_{0}=0, \mathscr{K}_{3}$, when $x_{0}=y_{3}=0$ and $\mathscr{K}_{4}$, when $x_{3}=y_{3}=0$. The Hilbert dimension of these ideals over the ring $\mathbb{C}\left[h_{0}, h_{1}, h_{2}\right]$ is found to be $\operatorname{six}^{1}$ and hence the global mobility of the 3-RPS-3-SPR S-PM.

$$
\overline{\operatorname{dim}} \mathscr{K}_{i}=6, \quad i=1,2,3,4 .
$$

\section{Conclusions and future work}

In this paper, the full-cycle mobility of a 3-RPS-3-SPR PM was elucidated to be six. The kinematic Jacobian matrix of the series-parallel manipulator was calculated with the help of screw theory and the result was extended to $n$-number of modules. Moreover, the methodology for the determination of the twist system of series-parallel manipulators was explained. The rank of the Jacobian matrix or the order of the twist system gives the local mobility of the S-PM. Global mobility was calculated as the Hilbert dimension of the ideal of the set of constraint equations. In the future, we intend to solve the inverse and direct kinematics using algebraic geometry concepts and to enlist all possible singularities of series-parallel mechanisms. Additionally, it is challenging to consider $n$-modules $(n>2)$ and to work on the trajectory planning of such manipulators as the number of output parameters is equal to six and lower than the number of actuated joints, which is equal to $3 n$.

Acknowledgements This work was conducted with the support of both the École Centrale de Nantes and the French National Research Agency (ANR project number: ANR-14-CE34-0008$01)$.

\section{References}

[1] Amine, S., Mokhiamar, O., Caro, S.: Classification of 3T1R parallel manipulators based on their wrench graph. ASME. J. Mechanisms Robotics. 9(1) (2017)

[2] Binaud, N., Cardou, P., Caro, S., Wenger, P.: The kinematic sensitivity of robotic manipulators to joint clearances. In: Conference : International Design Engineering Technical Conferences and Computers and Information in Engineering Conference, pp. 1371-1380 (2010)

\footnotetext{
${ }^{1}$ The pdf file of the Maple sheet with calculation of Hilbert dimension can be found here : https://www.dropbox.com/s/3bqsn45rszvgdax/Mobility3RPS3SPR.pdf?dl=0
} 
[3] Gallardo-Alvarado, J., Abedinnasab, M.H., Rodríguez-Castro, R., AguilarNájera, C.R.: Kinematics of a series-parallel manipulator with constrained rotations by means of the theory of screws. In: V.M. Petrova (ed.) Advances in Engineering Research. Nova Science Publishers, NewYork (2016)

[4] Gallardo-Alvarado, J., Pérez-González, L., Ruiz-Mondragón, G., RojasGarduño, H., Villagómez, A.T.: Mobility and velocity analysis of a limited-dof series-parallel manipulator. In: Conference : XVII COMRob 2015, Mexican Robotics Congress (2015)

[5] Hu, B.: Formulation of unified Jacobian for serial-parallel manipulators. Robotics and Computer-Integrated Manufacturing 30(5), 460 - 467 (2014)

[6] Hu, B., Lu, Y., Yu, J.J., Zhuang, S.: Analyses of inverse kinematics, statics and workspace of a novel 3-RPS-3-SPR serial-parallel manipulator. Open Mech. Eng. J. 6(Suppl1-M5), 65-72 (2012)

[7] Hunt, K.H.: Structural kinematics of in-parallel-actuated robot-arms. ASME. J. Mech., Trans., and Automation. 105(4), 705-712 (1983)

[8] Husty, M.L., Schröcker, H.P.: A Proposal for a New Definition of the Degree of Freedom of a Mechanism, pp. 109-117. Springer Netherlands, Dordrecht (2011)

[9] Joshi, S.A., Tsai, L.W.: Jacobian analysis of limited-DOF parallel manipulators. ASME, Mechanism Design J. 124(2), 254-258 (2002)

[10] Maraje, S., Nurahmi, L., Caro, S.: Operation modes comparison of a reconfigurable 3-PRS parallel manipulator based on kinematic performance. In: Proceedings of the ASME 2016 International Design Engineering Technical Conferences and Computers and Information in Engineering Conference, pp. 21-24 (2016)

[11] Murray, R.M., Sastry, S.S., Zexiang, L.: A Mathematical Introduction to Robotic Manipulation

[12] Nayak, A., Nurahmi, L., Wenger, P., Caro, S.: Comparison of 3-RPS and 3SPR parallel manipulators based on their maximum inscribed singularity-free circle. In: New Trends in Mechanism and Machine Science, pp. 121-130 (2017)

[13] Nurahmi, L., Caro, S.: Dimensionally homogeneous extended jacobian and condition number. In: The 2nd International Conference on Mechanical Engineering (ICOME 2015), pp. 3-5 (2015)

[14] Schadlbauer, J., Walter, D., Husty, M.: The 3-RPS parallel manipulator from an algebraic viewpoint. Mechanism and Machine Theory 75, 161 - 176 (2014)

[15] Zlatanov, D., Fenton, R.G., Benhabib, B.: A unifying framework for classification and interpretation of mechanism singularities. ASME, Mechanism Design J. 117(4), 566-572 (1995) 